\title{
CONTINUOUS DEPENDENCE OF SOLUTIONS OF OPERATOR EQUATIONS. I( $\left.{ }^{1}\right)$ \\ BY
}

ZVI ARTSTEIN

\begin{abstract}
Continuous dependence of the solutions of the operator equation $x=T x+z$ in a topological vector space is the main subject of the paper. We find sufficient and necessary conditions for the continuous dependence on the data $(T, z)$ or on a parameter. We do it for the space of all closed operators. Equivalent conditions for particular subfamilies are discussed. Among other families we deal with compact operators, compact perturbations of the identity, condensing operators and demicompact operators.
\end{abstract}

1. Basic ideas and main results. Many differential and integral equations as well as functional equations can be reduced to an operator equation $x=T x$ $+z$ where $x$ and $z$ are members of a topological vector space $X$ and $T$ is a (nonlinear in general) operator from $X$ to itself. Our main purpose is to analyze the dependence of the solutions of this equation on the operator $T$ and the forcing term $z$.

There are two related approaches of analyzing the continuous dependence of solutions of the operator equations. One approach is to consider parameterized families $T_{\lambda}$ of operators, where $\lambda$ belongs to a topological space, and investigate the dependence of the solutions on the parameter $\lambda$. A second approach is to impose a topology on the collection of operators $T$ and then investigate the continuity of the solutions with respect to this topology. The second approach contributes also to the first one. Indeed, if the solution is continuous with respect to a certain topology on the collection of operators, then the continuity of the parameterization $\lambda \rightarrow T_{\lambda}$ implies the continuous dependence on the parameter.

A main goal of ours it to relax sufficient conditions that appear in the literature and hopefully get necessary conditions. In terms of topologies on the operators $T$, relaxing the conditions means imposing a smaller topology that still yields the continuous dependence. Necessary conditions might mean finding the smallest such topology. Now a difficulty arises: Under quite

Received by the editors September 23, 1974 and, in revised form, January 8, 1976.

AMS (MOS) subject classifications (1970). Primary 47-00; Secondary 47H99, 39A10, 45G99.

Key words and phrases. Continuous dependence on parameters, closed operators, compact operators, condensing operators, fixed points, ODE, FDE.

(') The research at Brown University was supported by the Office of Naval Research under NONR N0014-67-A-0191-0009 and the National Science Foundation under GP 28931 X2.

- American Mathematical Society 1977 
general circumstances there is no smallest topology on the collection of operators $T$ so that the solution will depend continuously on $T$ and the forcing term $z$. What can be done is to characterize those topologies with respect to which the continuous dependence holds. We do it, but we also proceed in a different direction. Instead of considering continuity with respect to a topology we discuss continuity with respect to a convergence space. In the class of convergence structures we are able to find the analogue of the smallest topology; it is the richest convergence structure with respect to which the continuous dependence holds. In this way we provide necessary conditions for the continuous dependence. For a discussion about the precise extent of the necessity of the conditions see $\$ 2$.

We are able to describe the necessary and sufficient conditions for the family of all closed operators. As a matter of fact, at this level of abstractness it is quite easy to find the conditions, they are a natural outcome of the setting and the structure of the problem. The novelty of the approach is that the conditions represent a hereditary property. Thus the restriction of the convergence structure to a subfamily of closed operators gives necessary and sufficient conditions for the particular subfamily. Not surprisingly, we find that known conditions for several distinct particular examples are equivalent to our conditions. Therefore we have a unified approach that generalizes, and sometimes improves, many results that appear in the literature for different and special classes. Some classes are compact operators, $\alpha$-condensing and demicompact operators. In all these cases we add to the existing theory the aspect of the necessity of the conditions.

We want to separate the problem of existence and uniqueness from the continuous dependence, and to concentrate on the latter. Without posing conditions that imply uniqueness, or without assuming uniqueness of the solution, we have a set $s(T, z)$ of solutions of the equation $x=T x+z$, and this set might be empty. In order to discuss the continuous dependence of the multivalued mapping $s$ on the data $(T, z)$ we consider two types of semicontinuity. The first, and the weaker one, we call $L u$-continuity, and it is essentially equivalent to the closedness of the graph of $s$. As we mentioned earlier, the sufficient and necessary conditions for the $L u$-continuity of $s$ are

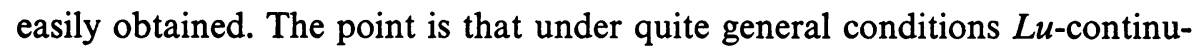
ity is equivalent to upper semicontinuity, and therefore in many cases we provide necessary and sufficient conditions for the upper semicontinuity. The relationship with actual continuity is that an upper semicontinuous mapping is continuous at any point where the value is a singleton. So when uniqueness holds, continuity is guaranteed.

The paper is organized as follows. In $\$ 3$ we construct an example of a family of operators on which there is no smallest topology with respect to 
which the continuous dependence holds. As we explained before, the inexistence of such a smallest topology leads us to use the convergence spaces; they are introduced in $\$ 4$. The richest convergence structure that yields the continuous dependence is discussed in $\$ 5$. In $\$ \S 6-8$ three particular classes are investigated. They are the collectively compact, collectively compact perturbations of the identity and collectively $\alpha$-condensing operators. A case where a smallest topology does exist is presented in $\$ 9$, and in $\S 10$ we discuss the case where we are interested only in sequential continuity; here the compact-open topology plays in a certain sense the role of the smallest topology. In the final section, we discuss another class of operators, the demicompact operators. Here the necessary and sufficient conditions are expressed in terms of the inverse operator, which is a multivalued operator.

The results show that it is more natural to deal with multivalued operators and equations of the form $x \in T x+z$. When we deal with the examples we often assume that $T$ is single-valued, mainly for the sake of simplifying notations. Most of these results can be generalized to the multivalued case.

AcKnowledgement. The author wishes to thank J. K. Hale for his help and encouragement.

2. The necessity of the conditions. When we examine the quite extensive literature on continuous dependence we notice that usually only sufficient conditions are provided. This is true both when abstract operator equations, as in the previous section, are discussed, or when particular systems such as ode's or integral equations are investigated. There is a very good reason for this fact. Usually the operator form of the equation is $x=T x$ and the continuous dependence in question is on the operator $T$. There is almost no hope to get reasonable necessary conditions for the dependence on $T$. The values of $T$ that are involved in "creating" the solutions of the equation are those near the fixed points. We may change $T$ a lot and still maintain the same set of fixed points, i.e., solutions. So a global necessary condition, say a convergence in the space of operators, cannot be deduced from the convergence of the solutions.

At this point we have two choices. We can try to find the best sufficient conditions, where "best" has a prespecified meaning (this approach was developed in Artstein [1975a]), or we can change the problem a bit and find necessary conditions for the new, but related, problem. In this paper we add a forcing term $z$ to the equation $x=T x$ and are successful in finding necessary conditions for the continuous dependence of solutions of $x=T x+z$ on $T$ and $z$.

A forcing term of this kind appears naturally in many differential and integral equations. Notably many (not all) of the sufficient conditions en- 
countered in the literature for the continuous dependence on $T$ are valid for the dependence on $(T, z)$ as well.

One possibility is still flexible, namely the choice of the collection on which $z$ ranges. The smaller the range of $z$ the better our theory is. For instance, if the integral form of an ordinary differential equation

$$
x(t)=\int_{a}^{t} f(x(s), s) d s+g(t)
$$

is considered, then the restriction $g(t)=$ constant provides a theory of continuous dependence on the vector field $f$ and the initial data $x(a)=g(a)$; and continuous dependence on $g(t)$ in the space of continuous functions is, in general, a stronger demand. In this paper we mainly let $z$ vary on the whole space $X$. In some particular examples we investigate the hierarchy of spaces of forcing terms that still guarantee the necessity of the conditions.

3. A smallest topology does not exist. We shall construct a family $\mathbb{Q}_{1}$ of operators $T$ such that there is no smallest topology among the topologies on the family with the property that the solution of

$$
x=T x+z
$$

is continuous in the pair $(T, z)$.

Let $X$ be an infinite dimensional Banach space. Let $\left\{w_{m}: m=1,2, \ldots\right\}$ be a sequence of vectors of unit norm, no subsequence of which converges. Let $y$ be a fixed vector and define

$$
T_{n, m} x=\exp \left(-m\left\|x-n^{-1} w_{m}\right\|\right) \cdot y .
$$

In this way $T_{n, m}: X \rightarrow X$ is a compact operator for every $n, m=1,2, \ldots$ Also the equation $x=T_{n, m} x+z$ has a solution for each $z$ in $X$. Our family $\mathcal{Q}_{1}$ of operators consists of the operators $T_{n, m}$ and the zero operator $\theta$ given by $\theta x=0$ for all $x$.

Let us fix $n_{0}$ and consider the topology on $\mathcal{Q}_{1}$ generated by one converging sequence, namely $T_{n_{0}, m} \rightarrow \theta$ as $m \rightarrow \infty$ (this is a metrizable topology). We claim that the solution of $(*)$ is continuous with respect to this topology. Indeed, if $x_{m}=T_{n_{0}, m} x_{m}+z_{m}$, the precompactness of the common range of $T_{n_{0}, m}$ implies that for every subsequence $\left\{x_{l}\right\}$ the sequence $T_{n_{0}, l} x_{l}$ has a converging subsequence $T_{n_{0}, k} x_{k}$; if, in addition, $z_{k} \rightarrow z$, the sequence $x_{k}$ converges. But then $T_{n_{0}, k} x_{k}$ converges to 0 . Therefore every subsequence of $\left\{x_{m}\right\}$ has a converging subsubsequence, with one limit $z$. Therefore $x_{m} \rightarrow z$, and the continuous dependence holds.

If there exists a smallest topology $\mathcal{T}$ with respect to which the continuous dependence holds, it should be smaller than the one in the preceding paragraph, in particular, $T_{n_{0}, m} \rightarrow \theta$ in $\mathcal{T}$ as $m \rightarrow \infty$ for every fixed $n_{0}$. The principle of iterated limits implies that the net $T_{n, m}$, directed by $(n, m)>(k$, 
$l$ ) if $n>k$ and $m>l$, converges to $\theta$ in $\mathscr{T}$ (see Kelley [1955, pp. 69, 74]). We shall see that the last convergence cannot hold. We notice that the net $w_{n, m}=w_{m} / n$ converges to 0 . Define $z_{n, m}$ by $z_{n, m}=w_{n, m}-T_{n, m} w_{n, m}$. Then on one hand $w_{n, m}$ is a solution of $x=T_{n, m} x+z_{n, m}$; on the other hand $z_{n, m} \rightarrow y$, and if $T_{n, m} \rightarrow \theta$ in $\mathcal{T}$ the limit of $w_{n, m}$, namely 0 , is a solution of $x=\theta x+y$, but it is not. This concludes the proof.

REMARK. The construction above is a paraphrase of the example given in Artstein [1975b, Appendix B].

4. Convergence spaces. Abstract spaces with convergence as the primitive structure were already introduced by Fréchet in 1906. In Kuratowski [1966] the reader can find a discussion on $L^{*}$ spaces, where the convergence of sequences is used. We want our spaces to include general topological spaces, and therefore we shall use as a primitive notion the convergence of nets. As a reference for the definition of nets, the properties of the convergence and the Moore-Smith convergence in topological spaces, we shall use J. L. Kelley's book [1955].

We say that a convergence structure is given for a set $V$ if to certain nets $\left\{v_{n} ; n \in N\right\}$ in $V$ (called the convergent nets) there corresponds an element $v$ in $V$, denoted by $\lim u_{n}$, so that the following conditions are fulfilled.

(a) If $v_{n}=v$ for every $n$ then $\lim v_{n}=v$.

(b) If $\lim v_{n}=v$ and $\left\{v_{m}\right\}$ is a subnet then $\lim v_{m}=v$.

(c) If $\left\{v_{n}\right\}$ does not converge to $v$ then a subnet of $\left\{v_{n}\right\}$ exists, no subset of which converges to $v$.

A set with a convergence structure on it is a convergence space. We shall use the notation $(V, \mathrm{lim})$ to indicate the convergence space when more than one convergence structure is imposed on the same set.

Not every convergence space is topological in the sense that the convergence structure coincides with the Moore-Smith convergence of a certain topology. A necessary and sufficient condition for a convergence space to be topological is that the "convergence of the iterated limit" property (see (d) in Kelley [1955, p. 74]) will hold.

If $V$ and $U$ are convergence spaces, the cartesian product $V \times U$ is a convergence space when we define $\lim \left(v_{n}, u_{n}\right)=(v, u)$ if $\lim v_{n}=v$ in $V$ and $\lim u_{n}=u$ in $U$.

A function $f: U \rightarrow V$ is continuous if $\lim u_{n}=u$ implies $\lim f\left(u_{n}\right)=f(u)$.

If $U_{1}$ is a subset of the convergence space $U$ then $U_{1}$ itself is a convergence space with respect to the restriction of the convergence structure on $U$. We say that $U_{1}$ is a subspace.

Sometimes more than one convergence structure is considered on the same set. Let $\lim ^{1}$ and $\lim ^{2}$ be two convergence structures on $U$. We say that $\lim ^{1}$ is richer than $\lim ^{2}$ if $v=\lim ^{2} v_{n}$ implies $v=\lim ^{1} v_{n}$; i.e., there are more (but not 
necessarily strictly more) convergence nets with respect to $\lim ^{1}$ than with respect to $\lim ^{2}$. If $\left(U, \lim ^{1}\right)$ and $\left(U, \lim ^{2}\right)$ are topological then $\lim ^{1}$ is richer than $\lim ^{2}$ if and only if the topology associated with $\lim ^{1}$ is smaller (= weaker, coarser, has less open sets) than the one associated with lim$^{2}$.

5. The continuity with respect to the space of closed operators. We shall formulate here our most general theorem. We shall find the richest convergence structure on a general space of closed operators, with respect to which the solution-set is $\mathrm{Lu}$-continuous (the concept will be defined below). As was explained in the introduction, the result in this generality is quite simple, its power is that in many particular situations the conditions are equivalent to more concrete conditions, and that $L u$-continuity implies upper semicontinuity or continuity.

Let $X$ be a topological vector space. A multivalued operator $T$ from $D(T) \subset X$ into $X$ is closed if Graph $T=\{(x, y): y \in T x\}$ is a closed subset of $X \times X$.

We denote by $C$ the collection of all closed multivalued operators from $X$ to itself. For $T \in \mathcal{C}$ and a vector $z \in X$ we denote by $s(T, z)$ the set of solutions of the equation

$$
x \in T x+z
$$

(here $T x+z=\{y+z: y \in T x\}$, and $x$ is a solution if the relation (*) holds). Since we do not assume existence or uniqueness the $\operatorname{set} s(T, z)$ might be empty or contain many elements. Notice, however, the closedness of $T$ implies that $s(T, z)$ is closed.

Definition 5.1. Let $\left\{T_{n} ; n \in N\right\}$ be a net in $\mathcal{C}$; we say that the net converges to the operator $T_{0}$ in $\mathcal{C}$ if whenever $\left\{T_{m} ; m \in M\right\}$ is a subnet, $y_{m} \in T_{m} x_{m}$ and $\left(x_{m}, y_{m}\right) \rightarrow\left(x_{0}, y_{0}\right)$ in $X \times X$ then $y_{0} \in T_{0} x_{0}$. We then write $T_{0}=\operatorname{Lum} T_{n}$.

Notice that the limit concept Lum is quite weak. For instance, if for no subnet $\left\{T_{m}\right\}$ and pairs $y_{m} \in T_{m} x_{m}$ the convergence $\lim \left(x_{m}, y_{m}\right)$ holds, then $\left\{T_{n}\right\}$ converges to any element in $\mathcal{C}$. Also the limit $\operatorname{Lum} T_{n}$ is not unique, but there is a unique limit with a minimal graph, consisting of all pairs $\lim \left(x_{m}, y_{m}\right)$ given as above.

Proposition 5.2. The space ( $($, Lum) is a convergence space.

Proof. We have to verify the validity of conditions (a), (b) and (c) in $\$ 4$. Property (a) is implied by the closedness of $T$. Property (b) follows from the fact that a subnet of a subnet is a subnet. Suppose now that $\left\{T_{n}\right\}$ does not converge to $T$. Then a subnet $\left\{T_{m}\right\}$ exists, and pairs $\left(x_{m}, y_{m}\right)$ such that $y_{m} \in T_{m} x_{m}, \lim \left(x_{m}, y_{m}\right)=(x, y)$ but $y \notin T x$. No subnet of $T_{m}$ converges then to $T$. This completes the proof. 
A multivalued function $f$ from the convergence space $U$ to the convergence space $W$ is Lu-continuous if $\lim u_{n}=u, w_{n} \in f\left(u_{n}\right)$ and $\lim w_{n}=w$ imply that $w \in f(u)$. If $U$ and $W$ are topological spaces then the $L u$-continuity of $f$ is equivalent to the closedness of the graph of $f$.

TheOrem 5.3. Let $\mathbb{Q}$ be a subspace of $\mathcal{C}$. The multivalued function $s(T, z)$ from the product space (Q, Lum) $\times X$ into $X$ is Lu-continuous. Furthermore, the convergence structure Lum is the richest convergence structure on $\mathbb{Q}$ with respect to which $s(T, z)$ is Lu-continuous.

Proof. Suppose that $\left\{\left(T_{n}, z_{n}\right)\right\}$ converges to $\left(T_{0}, z_{0}\right)$ in $\mathcal{Q} \times X$ and that $\left\{x_{n}\right\}$ converges to $x_{0}$. We have to show that if $x_{n} \in T_{n} x_{n}+z_{n}$ for every $n$, then $x_{0} \in T_{0} x_{0}+z_{0}$. Equivalently, if $x_{n}-z_{n} \in T_{n} x_{n}$ for every $n$, then $x_{0}-$ $z_{0} \in T_{0} x_{0}$. But this is implied directly from the convergence of $\left\{\left(x_{n}-z_{n}, x_{n}\right)\right\}$ to $\left(x_{0}-z_{0}, x_{0}\right)$ and the convergence of $\left\{T_{n}\right\}$ to $T_{0}$ (see Definition 5.1). Suppose now that we have another convergence structure on $\mathcal{Q}$, say lim, and suppose that Lum is not richer than lim. Then for a certain net $\left\{T_{n}\right\}$ the relation $\lim T_{n}=T_{0}$ holds but $\operatorname{Lum} T_{n}=T_{0}$ is not true. The latter means that for a subnet $\left\{T_{m}\right\}$ and a net $\left\{\left(x_{m}, y_{m}\right)\right\}$ the relations $y_{m} \in T_{m} x_{m}$ hold, and $\lim \left(x_{m}, y_{m}\right)=\left(x_{0}, y_{0}\right)$ but $y_{0} \notin T_{0} x_{0}$. If we now define $z_{m}=x_{m}-y_{m}$, then $z_{m} \rightarrow z_{0}=x_{0}-y_{0}$. Summing up, $\lim T_{m}=T_{0}, \lim z_{m}=z_{0}, \quad x_{m} \in$ $s\left(T_{m}, z_{m}\right)$ and $\lim x_{m}=x_{0}$, but $x_{0} \notin s\left(T_{0}, z_{0}\right)$. This shows that $s(T, z)$ is not $\mathrm{Lu}$-continuous with respect to lim. This completes the proof.

A natural question is whether a convergence space ( $Q$, Lum) is topological, and if not, whether a smallest topology that yields the continuous dependence exists. We have already seen in $\$ 3$ that such a smallest topology does not exist in general. We now can say more.

Proposition 5.4. Let $\mathbb{Q}$ be a subset of $\mathcal{C}$. If $(\mathcal{Q}, \mathrm{Lum})$ is not topological then there is no smallest topology on $\mathbb{Q}$ with respect to which $s(T, z)$ from $\mathbb{Q} \times X$ into $X$ is Lu-continuous.

Proof. Let $\mathcal{T}$ be a topology on $\mathbb{Q}$ and suppose that $s(T, z)$ is $L u$-continuous as a multivalued function from $(Q, \mathcal{G}) \times X$ into $X$. Every topological space is also a convergence space and therefore Theorem 5.3 implies that Lum on $\mathcal{Q}$ is richer than the Moore-Smith convergence with respect to $\mathcal{T}$. If ( $Q$, Lum) is not topological then Lum is strictly richer than the convergence in $\mathcal{T}$; i.e., there is a net $\left\{T_{n} ; n \in N\right\}$ in $\mathbb{Q}$ and an element $T_{0} \in \mathbb{Q}$ such that Lum $T_{n}=T_{0}$ but $\left\{T_{n}\right\}$ does not converge to $T_{0}$ in $\mathcal{T}_{\text {. Define a topology } \mathcal{T}_{1}}$ on $\mathscr{Q}$ as follows. If $T \neq T_{0}$ then $T$ is an isolated point of $\mathcal{T}_{1}$ (i.e., $\{T\}$ is an open set). A neighborhood of $T_{0}$ is a collection $Q$ such that $\left\{n: T_{n} \in Q\right\}$ is residual in $N$. Clearly, the Lum convergence is richer than the convergence with respect to $\mathcal{T}_{1}$, and therefore $s(T, z)$ is $L u$-continuous on $\left(Q, \mathcal{T}_{1}\right) \times X$. On 
the other hand $\mathscr{T}$ is not smaller than $\mathscr{T}_{1}$; indeed, $\left\{T_{n}\right\}$ converges in $\mathscr{T}_{1}$ but not in $\mathcal{T}$. This completes the proof.

As the converse of Proposition 5.4 is concerned, then obviously if (Q, Lum) is topological then the topology is the desired smallest topology. The answer to the question whether ( $Q$, Lum) is topological depends, of course, on $\mathbb{Q}$. For the collection $\mathcal{C}$ of all the closed operators we can state that $(\mathcal{C}, \mathrm{Lum})$ is topological if and only if $X$ is locally compact. We omit the proof; it is technical and involves a careful checking of condition (d) in Kelly [1955, p. 74] (see \$4).

We conclude this section by examining the application of Theorem 5.3 to parameterized families of operators. Let $\Lambda$ be a convergence space, the space of parameters. A function $p: \Lambda \rightarrow \circlearrowright$ is called a parameterization, and we denote $p(\lambda)$ either by $T_{\lambda}$ or $T(\lambda)$. Given a parameterization $p$ we denote $s(\lambda, z)=s\left(T_{\lambda}, z\right)$, i.e., $s(\lambda, z)$ is the multivalued function that associates with $(\lambda, z) \in \Lambda \times X$ the set of solutions of $x \in T_{\lambda} x+z$. We are interested in the continuity properties of $s(\lambda, z)$.

Proposition 5.5. Let $p: \Lambda \rightarrow \bigodot$ be a parameterization. A necessary and sufficient condition for $s(\lambda, z)$ to be Lu-continuous is that $p: \Lambda \rightarrow(\mathcal{C}$, Lum $)$ is continuous.

Proof. The function $s(\lambda, z)$ is the composition of the $L u$-continuous mapping $s(T, z)$ and the function $p$; therefore if the latter is continuous then the composition is $L u$-continuous (as it is easy to check). This proves the sufficiency. To prove the necessity suppose that $p$ is not continuous; then a convergent net $\left\{\lambda_{n} ; n \in N\right\}$ exists with limit $\lambda_{0}$ in $\Lambda$ but such that $\left\{T\left(\lambda_{n}\right)\right\}$ does not converge to $T\left(\lambda_{0}\right)$. Consider the collection $\left\{T\left(\lambda_{n}\right): n \in N\right\} \cup$ $\left\{T\left(\lambda_{0}\right)\right\}$ as a topological space where $\left\{T\left(\lambda_{n}\right)\right\}$ is an open set if $T\left(\lambda_{n}\right) \neq T\left(\lambda_{0}\right)$ and a neighborhood of $T\left(\lambda_{0}\right)$ is a residual set of the net $\left\{T\left(\lambda_{n}\right)\right\}$. Then the convergence structure Lum is not richer than the convergence with respect to this topology. Therefore $s(T, z)$ is not $L u$-continuous with respect to this topology (Theorem 5.3) and hence $s(\lambda, z)$ is not $L u$-continuous.

Notice that if the parameter set $\Lambda$ is a topological space then the continuity of the mapping $p: \Lambda \rightarrow(\mathcal{C}$, Lum $)$ is equivalent to the closedness of $\{(\lambda, x, y)$ : $\left.y \in T_{\lambda} x\right\}$ in $\Lambda \times X \times X$. Therefore the closedness of this set is a necessary and sufficient condition for the $L u$-continuity of $s(\lambda, z)$.

6. Collectively compact operators. For simplicity we shall work with operators which are single valued.

If $T$ is an operator with domain $D(T)$ in the topological vector space $X$, and if $A$ is a subset of $X$ we write $T A$ for $\{T x: x \in D(T) \cap A\}$. Recall that a subset $B$ of $X$ is bounded if for every neighborhood $Q$ of the origin there exists a number $r>0$ such that $B \subset r Q$. The space $X$ is locally bounded if 
there is a bounded neighborhood of the origin, see Köthe [1969, pp. 152, 159]. A topological vector space is locally bounded if and only if its topology can be given by a quasi-norm, see Köthe [1969, p. 159].

An operator $T$ is compact if $T B$ is precompact (i.e., has compact closure) for every bounded set $B$. A collection $थ$ of operators is collectively compact if $\cup_{T \in \mathcal{Q}} T B$ is precompact for every bounded set $B$.

An operator $T$ is compact-range if $T X$ is precompact. A collection $थ$ of operators is collectively compact-range if $\cup_{T \in \mathcal{Q}} T X$ is precompact.

Many operators encountered in the literature are either compact or compact-range. The compactness is very useful in proving existence of solutions of the operator equation $x=T x+z$. Collectively compact families were also discussed in the literature, see Anselone [1971] and the references therein. Continuous dependence for collectively compact operators was also discussed, either in an abstract form or for particular families. Some references are Costello [1971], Driver [1965], Gaines [1969], Grimm [1971], Hale and Cruz [1970], Ingram [1972], Klaasen [1975], Kurzweil and Vorel [1957], Neustadt [1970] and Wenska [1972].

The main result of this section is to find, for collectively compact families of operators, an equivalent condition for the Lum convergence, hence a new formulation for the necessary and sufficient condition given in Theorem 5.3. The respective modification for the dependence on parameters will also be shown. In addition, we shall see that for these families the $L u$-continuity is actually upper semicontinuity, hence continuity in the case of uniqueness.

The theory will, in general, be valid for compact-range operators in any space, while for compact operators we usually have to assume that the space is locally bounded. We work with closed operators, but we actually know more as the following lemma shows.

Lemma 6.1. A closed and compact-range operator is continuous. If the space is locally bounded then a closed and compact operator is continuous.

Proof. Let $\left\{x_{k}\right\}$ be a net in $D(T)$ which converges to $x$. We have to show that $T x_{k} \rightarrow T x$. Each subnet of $\left\{T x_{k}\right\}$ has a converging subsubnet (by the compactness property) and the closedness implies that the limit is $T x$. But there is a unique limit, so $\left\{T x_{k}\right\}$ itself converges.

DefinItion 6.2. The net $\left\{T_{k} ; k \in K\right\}$ of continuous operators, with domains $D\left(T_{k}\right)$, converges continuously to the operator $T$ if whenever $\left\{T_{m}\right\}$ is a subnet, $x_{m} \in D\left(T_{m}\right)$ and $\lim x_{m}=x$, then $x \in D(T)$ and $\lim T_{m} x_{m}=T x$. The convergence structure will be denoted CC (for continuous convergence).

Proposition 6.3. Let $F$ be a collection of continuous operators on X.Then $(F$, $\mathrm{CC})$ is a convergence space. 
Proof. Condition (a) in $\$ 4$ follows from the continuity. Condition (b) is built in the definition of CC. It is also simple to check (c).

We shall now see that for the families of operators treated in this section Definition 6.2 is equivalent to Definition 5.1.

THEOREM 6.4. Let $U$ be a collectively compact-range family of closed (hence continuous) operators defined on a topological vector space $X$. The convergence structure Lum is identical on थ with the convergence structure CC. If $X$ is locally bounded then the equivalence of Lum and CC holds also for collectively compact operators.

Proof. It is clear that if $T_{k} \rightarrow T$ in CC then $T_{k} \rightarrow T$ in Lum. Suppose now that $T_{k} \rightarrow T$ in Lum. Let $\left\{T_{m}\right\}$ be a subnet, $x_{m} \in D\left(T_{m}\right)$ and $\lim x_{m}=x$. Since $\mathscr{Q}$ is collectively compact-range (respectively $\left\{x_{m}\right\}$ is eventually bounded and $\mathcal{Q}$ is collectively compact) it follows that the elements $T_{k} x_{m}$ are in a compact set, and therefore the net $\left\{T_{m} x_{m}\right\}$ has a convergent subnet $\left\{T_{n} x_{n}\right\}$. The convergence Lum $T_{m}=T$ implies that $\lim T_{n} x_{n}=T x$ and, in particular, $x \in D(T)$. Since the limit of any convergent subnet of $T_{m} x_{m}$ is $T x$ it follows that $\lim T_{m} x_{m}=T x$. This completes the proof.

For the dependence on parameters we have the following modification (compare §5).

THEOREM 6.5. Let $थ$ be a collectively compact-range subfamily of $\mathcal{C}$. Let $p$ : $\Lambda \rightarrow$ थ be a parameterization. The mapping $p: \Lambda \rightarrow(\mathcal{Q}, \mathrm{CC})$ is continuous if and only if $T_{\lambda} x$ is continuous in the two variables $(\lambda, x)$. The same is true for a collectively compact family if $X$ is locally bounded.

Proof. Let $\lambda_{n} \rightarrow \lambda_{0}$ in $\Lambda$. If $\left\{T\left(\lambda_{m}\right)\right\}$ is a subnet of $\left\{T\left(\lambda_{n}\right)\right\}$ and $x_{m} \rightarrow x_{0}$ then the joint continuity of $T_{\lambda} x$ implies that $T_{\lambda_{m}} x_{m} \rightarrow T_{\lambda_{0}} x_{0}$, which proves that $\lambda \rightarrow T_{\lambda}$ is continuous. Conversely, if $\lambda \rightarrow T_{\lambda}$ is continuous, then $\lambda_{n} \rightarrow \lambda_{0}$, $x_{n} \rightarrow x_{0}$ implies $T_{\lambda_{n}} x_{n} \rightarrow T_{\lambda_{0}} x_{0}$, which means that $T_{\lambda} x$ is continuous in the pair $(\lambda, x)$.

We now turn to the properties of the set of solutions of the equation $x=T x+z$. As in the previous section we denote this set by $s(T, z)$.

Proposition 6.6. Let $T$ be a closed and compact range operator. Then for every $z$ the set $s(T, z)$ is compact. If $T$ is closed and compact then the intersection $s(T, z) \cap B$ with any closed bounded set $B$ is compact.

Proof. The set $s(T, z)$ (respectively, $s(T, z) \cap B)$ is contained in the precompact set $T X+z$ (respectively $T B+z$ ) and hence precompact. The continuity of $T$ (see Lemma 6.1) implies that $s(T, z)$ (respectively $s(T$, z) $\cap B$ ) is closed, hence compact.

The multivalued function $f$ from the convergence space $U$ to the topologi- 
cal space $W$ is upper semicontinuous if $\lim u_{n}=u$ in $U$ and $Q$ is an open neighborhood of $f(u)$, then the set of indices $n$ such that $f\left(u_{n}\right) \subset Q$ is residual (i.e., an $n_{0}$ exists so that $f\left(u_{n}\right) \subset Q$ for $n \geqslant n_{0}$ ). If both $U$ and $W$ are topological then the upper semicontinuity of $f$ is equivalent to the condition $\{u: f(u) \subset Q\}$ is open for any open $Q$ in $W$. It is easy to check that if $f\left(u_{0}\right)$ contains only one element $w_{0}$ then the upper semicontinuity implies that $u_{n} \rightarrow u$ and $w_{n} \in f\left(u_{n}\right)$ imply $w_{n} \rightarrow w_{0}$. In particular, if $f$ is single valued and upper semicontinuous it is continuous.

Proposition 6.7. Let $थ$ be a collectively compact-range family of closed operators. The Lu-continuity of $s(T, z):$ U $\times X \rightarrow X$ (with respect to a certain convergence structure on $Q$ ) implies its upper semicontinuity. If $X$ is locally bounded, then for a collectively compact family $\mathcal{Q}$, the Lu-continuity of $s(T, z) \cap B:$ थ $\times X \rightarrow X$ for a closed bounded $B$ implies that $s(T, z) \cap B$ is upper semicontinuous.

Proof. Suppose $\left(T_{n}, z_{n}\right) \rightarrow\left(T_{0}, z_{0}\right)$. Let $R$ be the compact set in $X$ that contains $T X$ for all $T$ in $Q$. Suppose that for a certain open set $Q$ which contains $s\left(T_{0}, z_{0}\right)$ a subnet $\left(T_{m}, z_{m}\right)$ exists with $y_{m} \in s\left(T_{m}, z_{m}\right)$ but $y_{m} \notin Q$. Each $y_{m}$ has the form $r_{m}+z_{m}$ where $r_{m} \in R$; therefore a convergent subnet of $\left\{y_{m}\right\}$ exists. On one hand the limit does not belong to $Q$, but on the other hand the $L u$-continuity implies that the limit belongs to $s\left(T_{0}, z_{0}\right) \subset Q$. The contradiction shows that such a subnet $\left\{y_{m}\right\}$ does not exist and therefore $s(T, z)$ is upper semicontinuous. In order to prove the second statement we just repeat the same argument with $R$ being the common range of $T B$.

The combination of Theorem 6.4 and Proposition 6.7 with Theorem 5.3 gives us the following result.

THEOREM 6.8. Let थ be a collectively compact-range family of closed operators. The convergence structure $\mathrm{CC}$ on $\mathcal{Q}$ is the richest convergence structure on थ with respect to which $s(T, z)$ : $\mathcal{Q} \times X \rightarrow X$ is upper semicontinuous. If $X$ is locally bounded then for a collectively compact family थ the convergence $\mathrm{CC}$ is the richest convergence structure with respect to which $s(T, z) \cap B:$ U $\times X \rightarrow X$ is upper semicontinuous. (The upper semicontinuity can be replaced by continuity in case $s(T, z)$ is single valued.)

The combination of Theorem 6.5 and Proposition 6.7 with Proposition 5.5 yields the following result.

THEOREM 6.9. Let $\Lambda$ be a convergence space of parameters. For each $\lambda$ let $T_{\lambda}$ be a closed operator so that the family $\left\{T_{\lambda}: \lambda \in \Lambda\right\}$ is collectively compactrange (respectively collectively compact and $X$ is locally bounded). Then $s(\lambda, z)$ (respectively $s(\lambda, z) \cap B$ for every closed bounded $B$ ) is upper semicontinuous if and only if $T_{\lambda} x$ is continuous in $\lambda$ and $x$ simultaneously. 
We have found that continuous convergence on a family of collectively compact operators is the richest structure with respect to which the (upper) continuous dependent holds. It is the richest structure with respect to another property as the following result shows. Let $U$ be a family of continuous operators. We say that a topology or a convergence structure on $\mathcal{U}$ is jointly continuous if the mapping $(T, x) \rightarrow T x$ from $थ \times X$ in $X$ is continuous.

Proposition 6.10. Let $थ$ be a family of continuous operators. The convergence structure $\mathrm{CC}$ is the richest jointly continuous structure.

Proof. The joint continuity of $\mathrm{CC}$ is implied directly from the definition. It is also clear that a net which converges in a jointly continuous structure satisfies Definition 6.2. This completes the proof.

Proposition 6.10 is a step toward the characterization of topologies (or convergence structures), on a space of collectively compact or compact-range operators, with respect to which the continuous dependence holds. Indeed, the solution set $s(T, z)$ is upper semicontinuous if and only if the topology on the elements $T$ is jointly continuous. This follows from Theorem 6.8 and Proposition 6.10. For a rigorous treatment of jointly continuous topologies and the existence or the nonexistence of a smallest jointly continuous topology see Arens [1946]. The analysis of the continuous dependence for a particular family of equations, namely Volterra integral equations, was done in Artstein [1975b].

7. Collectively compact perturbations of the identity. In the last section we found that the Lum convergence is equivalent to continuous convergence for collectively compact or compact-range operators. We shall see that the same conclusion holds for the almost counterpart of compact operators: compact perturbations of the identity.

An operator $T$ is a compact (respectively compact-range) perturbation of the identity if $T=I-K$ with $K$ being compact (respectively compactrange). Equivalently $T$ is a compact perturbation of the identity if $I-T$ is compact. A family $\mathcal{Q}$ of operators is collectively compact (respectively compact-range) perturbation of the identity if $\{I-T: T \in U\}$ is collectively compact (respectively compact-range).

The definitions of the Lum and the CC convergence are given in Definitions 5.1 and 6.2 respectively.

Lemma 7.1. A net $\left\{T_{k}\right\}$ converges continuously to $T$ if and only if $\left\{I-T_{k}\right\}$ converges continuously to $I-T$.

Proof. Since $T=I-(I-T)$ it is enough to prove only one side of the implication. Suppose then that $T_{k} \rightarrow T$. Let $\left\{I-T_{m}\right\}$ be a subnet of $\{I-$ $\left.T_{k}\right\}$ and let $x_{m} \in D\left(I-T_{m}\right)=D\left(T_{m}\right)$. If $\lim x_{m}=x$ then the continuous 
convergence of $\left\{T_{m}\right\}$ implies that $x \in D(T)=D(I-T)$ and $\lim T_{m} x_{m}=$ $T x$; i.e., $\lim \left(x_{m}-T_{m} x_{m}\right)=x-T x$. Therefore $I-T_{k} \rightarrow I-T$.

Lemma 7.2. Lum $T_{k}=T$ if and only if $\operatorname{Lum}\left(I-T_{k}\right)=\operatorname{Lum}(I-T)$.

Proof. Again, since $T=I-(I-T)$ it is enough to prove the "only if" part. Let Lum $T_{k}=T$. Let $\left(x_{k}, y_{k}\right) \in \operatorname{Graph}\left(I-T_{k}\right)$ with $\lim \left(x_{k}, y_{k}\right)=(x$, $y)$. Then $y_{k}=x_{k}-z_{k}$ where $z_{k} \in T_{k} x_{k}$ and $z_{k} \rightarrow x-y=z$. The relation Lum $T_{k}=T$ implies that $(x, z) \in$ Graph $T$, which means that $(x, x-z)=$ $(x, y) \in \operatorname{Graph}(I-T)$. This completes the proof.

Corollary 7.3 (of Lemma 7.1). An operator $T$ is continuous if and only if $I-T$ is continuous.

A direct proof is immediate.

THEOREM 7.4. Let $थ$ be a family of collectively compact-range perturbations of the identity. Assume that the operators in $थ$ are closed (hence continuous, see Lemma 6.1 and Corollary 7.3). The Lum convergence structure on $\mathscr{U}$ is identical with $\mathrm{CC}$. The same is true for collectively compact perturbations of the identity provided $X$ is locally bounded.

Proof. Theorem 6.4 tells us that Lum and CC are identical on $\{I-T$ : $T \in \mathcal{Q}$ \}. Lemmas 7.1 and 7.2 imply that the equivalence holds also for $थ$.

Although Lum and CC are equivalent on a family of collectively compact perturbations of the identity, the rest of $\$ 6$ cannot be generalized. For instance $s(T, z)$ is not necessarily compact as the equation $x=I x, I$ being the identity, shows.

8. Collectively condensing operators. Throughout this section we assume that the topological vector space $X$ is a complete metric space. We denote the metric by $d$. It is also assumed that the operators below are closed and single-valued.

If $B$ is a bounded set of $X$ then the Kuratowski measure $\alpha(B)$ of noncompactness is

$$
\alpha(B)=\inf \{\gamma>0: B \text { has a finite covering of sets with diameters }<\gamma\} .
$$

Obviously $\alpha(B)=0$ if and only if $B$ is precompact, i.e., has a compact closure. The measure $\alpha$ of noncompactness was introduced by Kuratowski [1930]. Some properties of $\alpha$ will be mentioned and used below. For more details see Darbo [1955] and Hale [1973]. Operators which are $\alpha$-condensing (the concept will be defined below) play an important role in studying infinite dimensional differential systems, functional and neutral equations. For a survey see Hale [1973], see also Szufla [1971]. A basic theorem that contains many former results on the continuous dependence on parameters of $\alpha$-con- 
densing operators was given by Hale [1974]. One of our main goals in this section is to see how Hale's theorem fits in the general continuous dependence results.

Here are some properties of the measure $\alpha$ of noncompactness. They are easily verified.

(i) $\alpha(B)=\alpha$ (closure $B)$.

(ii) $\alpha(A+B) \leqslant \alpha(A)+\alpha(B)$.

(iii) $A \subseteq B$ implies $\alpha(A) \leqslant \alpha(B)$.

An operator $T: X \rightarrow X$ is $\alpha$-condensing if $\alpha(T B)<\alpha(B)$ for every bounded $B$ with $\alpha(B)>0$ (equivalently $\alpha(T B) \geqslant \alpha(B)$ implies $\alpha(B)=0$ ). Notice that implicitly we assume that $T$ maps bounded sets into bounded sets. A collection $थ$ of operators is collectively $\alpha$-condensing if for every bounded $B$, $\alpha\left(\cup_{T \in \mathcal{Q}} T B\right) \geqslant \alpha(B)$ implies $\alpha(B)=0$.

An operator $T$ is $\alpha$-contraction if there exists a number $\gamma, 0<\gamma<1$, such that $\alpha(T B) \leqslant \gamma \alpha(B)$ for every bounded set $B$. A family $\mathscr{Q}$ of operators is collectively $\alpha$-contraction if a number $\gamma$ exists, $0<\gamma<1$ and $\alpha\left(\cup_{T \in \mathcal{Q}} T B\right) \leqslant$ $\gamma \alpha(B)$ for every bounded $B$.

LEMMA 8.1. Let $\left\{y_{k} ; k \in K\right\}$ be a net which is contained in a bounded set of $X$. Denote by $A_{k}$ the set $\left\{y_{m}: m \geqslant k\right\}$. If the numbers $\alpha\left(A_{k}\right)$ converge to zero then there exists a converging subnet $\left\{y_{n} ; n \in N\right\}$ of $\left\{y_{k}\right\}$.

Proof. For every $\varepsilon=1,2, \ldots$, there is a $k(\varepsilon)$ such that $\alpha\left(A_{k(\varepsilon)}\right) \leqslant \varepsilon^{-1}$. The set $A_{k(1)}$ can be covered by a finite number of balls of radius 1 . A cofinal subset $Y_{1}$ of the net $\left\{y_{k}\right\}$ is contined in one of the balls. The set $A_{k(2)}$ can be covered by a finite number of balls with radius $2^{-1}$, and a cofinal subset $Y_{2}$, such that $Y_{2} \subset Y_{1}$, is contained in one of the balls. Inductively, the set $A_{k(\varepsilon)}$ can be covered by a finite number of balls with radius $\varepsilon^{-1}$, and a cofinal set $Y_{\varepsilon}$, such that $Y_{\varepsilon} \subset Y_{\varepsilon-1}$, is contained in one of the balls. We define a subnet of $\left\{y_{k}\right\}$ as follows. The directed net consists of all the pairs $(k, \varepsilon)$ where $\varepsilon=1,2, \ldots$ and $y_{k} \in Y_{\varepsilon}$. The order is $(k, \varepsilon) \geqslant(l, \delta)$ if $k \geqslant l$ and $\varepsilon \geqslant \delta$. (Notice that the inequality $\varepsilon \geqslant \delta$ is between integers, while $k \geqslant l$ refers to the order in the directed set $K$.) We have to check that the collection of pairs $(k$, $\varepsilon)$ so defined is a directed set, but this follows easily from the cofinality of $Y_{\varepsilon}$ for each $\varepsilon$. Define now a net $\left\{y_{(k, \varepsilon)} ;(k, \varepsilon)\right\}$ by $y_{(k, \varepsilon)}=y_{k}$. This net is a subnet of $\left\{y_{k} ; k \in K\right\}$ since for every $\varepsilon$ the set $Y_{\varepsilon}=\left\{y_{m}: y_{m}=y_{(m, \varepsilon)}\right\}$ is cofinal. We claim that the net $\left\{y_{(k, \varepsilon)}\right\}$ is a Cauchy net. Indeed, our construction implies that all the sets $Y_{\varepsilon}$ for $\varepsilon \geqslant \varepsilon_{0}$ are contained in a fixed ball with radius $\varepsilon_{0}^{-1}$. A Cauchy net in a complete metric space has a limit. This completes the proof.

REMARK 8.2. The construction presented in the proof of Lemma 8.1 is, in essence, a standard diagonal process. If $\left\{y_{k}\right\}$ is a sequence then a converging subsequence can be found. 
LEMMA 8.3. $A$ closed and $\alpha$-condensing operator is continuous.

Proof. Since $X$ is metrizable it is enough to consider sequential continuity. Let $x_{k} \rightarrow x$ as $k \rightarrow \infty$. Then $\left\{x_{k}: k=1,2, \ldots\right\}$ is precompact: If $T$ is $\alpha$-condensing then $\left\{T x_{k}: k=1,2, \ldots\right\}$ is also a precompact set. By the closedness of $T$ every limit point of $\left\{T x_{k} ; k=1,2, \ldots\right\}$ is equal to $T x$. Therefore $\lim T x_{k}=T x$.

Similar to Theorem 6.4 we shall now see that for a collectively $\alpha$-condensing family the Lum convergence (see Definition 5.1 ) coincides with the CC convergence (see Definition 6.2).

THEOREM 8.4. Let $\mathcal{U}$ be a collectively $\alpha$-condensing set of closed (hence, continuous) operators. Then Lum $T_{k}=T$ in $\mathcal{Q}$ if and only if $\left\{T_{k}\right\}$ converges continuously to $T$.

Proof. Clearly the continuous convergence implies the convergence in Lum. Suppose now that $\operatorname{Lum} T_{k}=T$ and that $\left\{T_{m}\right\}$ is a subnet and $x_{m} \rightarrow x$. We have to show that $\lim T_{m} x_{m}=T x$. Denore $B_{l}=\left\{x_{m}: m \geqslant l\right\}$. The diameter of $B_{l}$ converges to zero and therefore $\alpha\left(B_{l}\right) \rightarrow 0$. (Without loss of generality $B_{l}$ is bounded for every l.) Since $\mathcal{U}$ is collectively $\alpha$-condensing, it follows that $\alpha\left(\cup_{T \in \mathcal{Q}} T B_{l}\right)$ converges to 0 with $l$. In particular, $\alpha\left(A_{l}\right) \rightarrow 0$ where $A_{l}=\left\{T_{n} x_{n}: n \geqslant l\right\}$. Therefore by Lemma 8.1 every subnet of $\left\{T_{m} x_{m}\right\}$ has a convergent subsubnet, and the relation Lum $T_{m}=T$ implies that the limit of this subsubnet has to be $T x$. Therefore the whole net $\left\{T_{m} x_{m}\right\}$ converges to $T x$. This completes the proof.

The next step in obtaining the generalization of Theorem 6.8 to condensing operators is to generalize Proposition 6.7. But unfortunately a full generalization was not obtained. We recall that $s(T, z)$ is the set of solutions of $x=T x+z$.

LEMMA 8.5. If $T$ is $\alpha$-condensing then for every closed bounded set $B$ the set $B \cap s(T, z)$ is compact.

Proof. Since $x \in s(T, z)$ means that $x=T x+z$, it follows that

$$
T(s(T, z) \cap B)=s(T, z) \cap B-z .
$$

From (ii) it follows now that

$$
\alpha(T(s(T, z) \cap B))=\alpha(s(T, z) \cap B-z)+\alpha(\{z\}) \geqslant \alpha(s(T, z) \cap B),
$$

and since $T$ is $\alpha$-condensing, it follows that $s(T, z) \cap B$ is precompact. The continuity of $T$ implies that $s(T, z)$ is closed. This completes the proof.

Proposition 8.6. Let $\mathcal{Q}$ be a collectively $\alpha$-condensing family of operators. Let $Z$ be a locally compact subset of $X$. If for a certain convergence structure on 
Q the mapping $s(T, z)$ is Lu-continuous then the mapping $s(T, z) \cap B$ : थ $\times Z \rightarrow X$ is upper semicontinuous.

Proof. Suppose $T_{k} \rightarrow T$ in $\mathscr{U}$ and $z_{k} \rightarrow z$ in $Z$. Suppose that for a subnet $\left\{\left(T_{m}, z_{m}\right)\right\}$ there are elements $x_{m} \in s\left(T_{m}, z_{m}\right)$ which are out of a fixed open set $Q$ which contains $s(T, z)$. Now

$\alpha\left(\left\{x_{k}\right\}\right)=\alpha\left(\left\{T_{k} x_{k}+z_{k}\right\}\right) \leqslant \alpha\left(\bigcup_{T \in \mathcal{Q}} T\left\{x_{k}\right\}\right)+\alpha\left(\left\{z_{k}\right\}\right)=\alpha\left(\bigcup_{T \in \mathcal{U}} T\left\{x_{k}\right\}\right)$.

The inequality follows from (ii) while $\alpha\left(\left\{z_{k}\right\}\right)=0$ from the local compactness of $Z$. Since $\mathcal{Q}$ is collectively $\alpha$-condensing, it follows that $\left\{x_{k}\right\}$ is precompact. By the $L u$-continuity of $s$ it follows that every convergent subnet of $\left\{x_{k}\right\}$ must converge to an element of $s(T, z)$; this contradicts the assumption that $x_{k} \notin Q$. This completes the proof.

I do not know if Proposition 8.6 holds if we drop the compactness requirement on $Z$. The proof does not go through because a convergent net might not be compact. If we assume that $\mathcal{U}$ is collectively $\alpha$-contraction we can drop this requirement.

Proposition 8.7. Let $थ$ be a collectively $\alpha$-contraction family of operators. If for a certain convergence structure on $\mathcal{Q}$, the mapping $s(T, z)$ is Lu-continuous, then for every closed and bounded $B$ the mapping $s(T, z) \cap B: \mathcal{Q} \times X \rightarrow$ $X$ is upper semicontinuous.

Proof. Suppose that $T_{k} \rightarrow T$ in $\mathscr{Q}$ and that $z_{k} \rightarrow z$ in $X$. Let $x_{k} \in$ $s\left(T_{k}, z_{k}\right) \cap B$; we have to show that $\left\{x_{k}\right\}$ converges to the compact set $s(T, z) \cap B$, but since $s$ is $L u$-continuous it is enough to show that a convergent subnet exists. Denote $A_{k}=\left\{x_{n}: n \geqslant k\right\}$. We claim that $\alpha\left(A_{k}\right)$ converges to zero. Since $A_{k}$ decreases in $k$ it follows from (iii) that $\alpha\left(A_{k}\right)$ decreases. Suppose that the limit is positive, say $\delta$. Denote $Z_{k}=\left\{z_{n}: n>k\right\}$. Since $\lim z_{n}=z$ it follows that $\lim \alpha\left(Z_{k}\right)=0$. Properties (ii) and (iii) together with the collectively $\alpha$-contraction property imply that

$$
\alpha\left(A_{k}\right) \leqslant \alpha\left(\bigcup_{T \in \mathscr{U}} T A_{k}\right)+\alpha\left(Z_{k}\right) \leqslant \gamma \alpha\left(A_{k}\right)+\alpha\left(Z_{k}\right)
$$

which implies $\delta(1-\gamma) \leqslant \alpha\left(Z_{k}\right)$. But if $\delta>0$ then $\alpha\left(Z_{k}\right)$ cannot approach zero, a contradiction. Thus $\alpha\left(A_{k}\right)$ does converge to zero, and by Lemma 8.1 a convergent subnet of $\left\{x_{k}\right\}$ exists. This completes the proof.

The combination of Propositions 8.6 and 8.7 with Theorems 8.4 and 5.4 gives the following result. Notice that a necessary condition is provided only for $\alpha$-contractions.

THEOREM 8.8. Let थ be collectively $\alpha$-condensing. Then for every closed bounded $B$ and locally compact $Z$ the mapping $s(T, z) \cap B:($ थ,$C C) \times Z \rightarrow X$ 
is upper semicontinuous. If $थ$ is collectively $\alpha$-contraction then $\mathrm{CC}$ is the richest convergence structure with respect to which $s(T, z) \cap B: \mathcal{U} \times X \rightarrow X$ is upper semicontinuous.

In trying to generalize Theorem 6.9 to condensing operators we encounter the same difficulty as before. If the convergence in the parameter space $\Lambda$ is characterized by sequences, then sequential continuity is enough, therefore the forcing terms $z_{k}$ are always in a compact set and the analogue of Theorem 6.9 holds. Compare Artstein [1976] and Hale [1974]. It is not clear what happens if $\Lambda$ is a general space.

9. A case where a smallest topology does exist: A uniform modulus of continuity. In general the Lum convergence on a space of closed operators is not topological (see §5) and consequently there is no smallest topology with respect to which the continuous dependence holds (see Proposition 5.4). We shall discuss here a case where there exists a smallest topology with respect to which the continuous dependence holds.

Suppose that $X$ is a metric space and denote the distance function by $d$. Let $T$ be a single-valued operator from $D(T) \subset X$ into $X$.

A modulus of continuity for $T$ at the point $x \in D(T)$ is a nondecreasing function $\mu:[0, \infty) \rightarrow[0, \infty]$, continuous at 0 with $\mu(0)=0$ so that

$$
d(T x, T y) \leqslant \mu(d(x, y))
$$

whenever $y \in D(T)$. It is clear that $T$ is continuous at $x$ if and only if $T$ has a modulus of continuity at $x$. A collection $\mathscr{U}$ of operators with a common domain $D$ has a uniform modulus of continuity at $x \in D$ if a certain $\mu$ is a modulus of continuity at $x$ for each $T$ in $\mathcal{Q}$.

Recall from Definition 6.2 that the net $\left\{T_{k}\right\}$ of continuous operators with a common domain $D$ converges continuously (i.e., in CC) to $T$ if whenever $\left\{T_{m}\right\}$ is a subnet and $\lim x_{m}=x$ in $D$ then $\lim T_{m} x_{m}=T x$.

LEMMA 9.1. Let $\mathcal{U}$ be a family of operators defined on the same domain D. Suppose that for each point $x$ in $D$ the collection थ has a uniform modulus of continuity at $x$. Then $\left\{T_{k}\right\}$ converges continuously in $\mathcal{Q}$ to $T$ if and only if $\lim T_{k} x=T x$ for every $x \in D$ holds.

Proof. Obviously the pointwise convergence is implied by the continuous convergence. In order to verify the "if" part let $\lim x_{m}=x$ and $\left\{T_{m}\right\}$ be a subnet. Let $\mu$ be the guaranteed uniform modulus of continuity at $x$. Then

$$
\begin{aligned}
d\left(T_{m} x_{m}, T x\right) & \leqslant d\left(T_{m} x, T x\right)+d\left(T_{m} x_{m}, T_{m} x\right) \\
& <d\left(T_{m} x, T x\right)+\mu\left(d\left(x_{m}, x\right)\right)
\end{aligned}
$$

and therefore $\lim d\left(T_{m} x_{m}, T x\right)=0$. This completes the proof. 
Proposition 9.2. Let $थ$ be a collection of operators with a common domain $D$ and suppose that at every $x \in D$ the family $Q$ has $a$ uniform modulus of continuity. Then the convergence structure $\mathrm{CC}$ on $थ$ is topological. Furthermore the topology is the weak topology generated by the functionals $x: T \rightarrow T x$ for $x \in D$, i.e., a base for the topology is the collection of sets $\cap_{i=1}^{k}\left\{T: T x_{i} \in O_{i}\right\}$ where $x_{1}, \ldots, x_{k}$ is a finite collection of elements in $D$ and $O_{1}, \ldots, O_{k}$ are open sets in $X$.

Proof. The convergence of $\left\{T_{k}\right\}$ to $T$ in the weak topology generated by the functionals $x(T)=T x$ is equivalent to the pointwise convergence of $T_{k}$ to $T$, i.e., to $\lim T_{k} x=T x$ for every $x$. Under the assumptions of the proposition, the pointwise convergence is equivalent by Lemma 9.1 to the continuous convergence.

The application of Proposition 9.2 is immediate. Suppose that the collection $\mathscr{U}$ in the proposition happens to be collectively compact or compactrange, or collectively $\alpha$-condensing, or $\alpha$-contraction; then the results in $\S \S 6-8$ could be routinely reformulated in terms of the topology given in 9.2 rather than in terms of the continuous convergence. Compare, in particular, Propositions 6.6 and 6.7, Theorem 6.8, Proposition 8.6 and Theorem 8.8. Another interpretation is the following. Suppose the family $\mathscr{U}$ is given in a parameterized form, i.e., $T_{\lambda}$ for $\lambda$ in a convergence space. Then the continuity of $\lambda \rightarrow T_{\lambda}$, where $\left\{T_{\lambda}\right\}$ is endowed with the topology of pointwise convergence, is equivalent to the continuity of $T_{\lambda} x$ in $\lambda$ for every fixed $x$. Under the additional conditions of collectively compact or collectively condensing, for instance, the continuity of $T_{\lambda} x$ in $\lambda$ is therefore a necessary and sufficient condition for the continuous dependence. The difference between this condition and the other conditions discussed in the paper is that here the test for continuous dependence is given in the form of checking certain functionals from the parameter set into the vector space, i.e., $\lambda \rightarrow T_{\lambda} x$. Compare Artstein [1975a].

10. Metrizable parametrizations and sequential continuity. The continuous convergence of the operators is in many cases a necessary and sufficient condition for the continuous dependence of the solution set (see \$§6-8). In general the continuous convergence is not topological, i.e., there is no topology on the operators whose convergent nets are identical with the nets that converge in $\mathrm{CC}$. This situation changes if only convergent sequences are considered. There is a topology on the operators whose convergent sequences are identical with the sequences that converge in $\mathrm{CC}$. We shall find below that the compact-open topology is such a topology.

Our interest in the sequential convergence of the operators is motivated by the following situation. Let $\Lambda$ be the parameter set and assume that it is a 
metric space. Let $T_{\lambda}(\lambda \in \Lambda)$ be a collection of operators from $X$ to itself. We assume that $X$ is a metric space. Denote by $s(\lambda, z)$ the set of solutions of $x=T_{\lambda} x+z$. We have found that a necessary and sufficient condition for the $L u$-continuity of $s(\lambda, z)$ is that the mapping $\lambda \rightarrow T_{\lambda}$ is continuous with respect to the Lum convergence of the operators. In particular cases considered in $\$ \S 6-8$ the $L u$-continuity is reduced to upper semicontinuity while the Lum is identical with $\mathrm{CC}$, the continuous convergence. Therefore the necessary and sufficient condition is that $\left\{\lambda_{n}\right\}$ converges to $\lambda$ implies $\left\{T_{\lambda_{n}}\right\}$ converges continuously to $T_{\lambda}$. In our case, where $\Lambda$ and $X$ are metric spaces, the sequential continuity is enough, i.e., only convergent sequences $\left\{T_{n}\right.$; $n=1,2, \ldots\}$ have to be considered.

LEMMA 10.1. The multivalued function $s(\lambda, z)$ is upper semicontinuous if and only if it is sequentially upper semicontinuous, i.e., if whenever a sequence $\left\{\left(\lambda_{n}\right.\right.$, $\left.\left.z_{n}\right) ; n=1,2, \ldots\right\}$ converges to $(\lambda, z)$ and $x_{n} \in s\left(\lambda_{n}, z_{n}\right)$, then $\left\{x_{n}\right\}$ converges to $s(\lambda, z)$.

The proof is similar to the standard proof that in metric spaces sequential continuity is equivalent to continuity.

Proposition 10.2. Suppose that a necessary and sufficient condition for the upper semicontinuity of $s(\lambda, z)$ is that the mapping $\lambda \rightarrow T_{\lambda}$ will be continuous with respect to $\mathrm{CC}$. Then the sequential continuity of this mapping is also necessary and sufficient.

Proof. The necessity is obvious. If $\lambda \rightarrow T_{\lambda}$ is sequentially continuous with respect to $C C$ then the assumption implies that $s(\lambda, z)$ is sequentially upper semicontinuous, and by Lemma 10.1 it is upper semicontinuous. This complete the proof.

REMARK 10.3. A different proof could be given by arguing that the sequential continuity of $\lambda \rightarrow T_{\lambda}$ implies its continuity. This is true in the present case. In general if $f: M \rightarrow W$ is a function from a metric space to a convergence space, it is not true that sequential continuity implies continuity. (It is, of course, true if $W$ is topological too.)

In view of Proposition 10.2 the sequential continuity of $\lambda \rightarrow T_{\lambda}$ with respect to $\mathrm{CC}$ is the necessary and sufficient condition. As was noted in the beginning, we shall see that the convergent sequences of $\mathrm{CC}$ are identical to the convergent sequences of the compact-open topology. Therefore the continuity of $\lambda \rightarrow T_{\lambda}$ with respect to the compact-open topology (and now it is the usual continuity between topological spaces) is the sufficient and necessary condition for the upper semicontinuity of the solution set.

The basic fact, that the continuously convergent sequences coincide with the convergent sequences of the compact-open topology, is well known and 
appears in textbooks. However, for completeness we shall give the definition of the compact-open topology and a sketch of the proof.

Let $U$ be a collection of continuous functions from the metric space $X$ into the space $Y$. For any open set $Q$ in $Y$ and a compact set $K$ in $X$ let $C(K, Q)$ denote the collection of functions $T$ in $\mathcal{Q}$ such that $T K \subset Q$. The collection of sets $C(K, Q)$ is a base for the compact-open topology on $\mathcal{Q}$. See Kelley [1955].

Recall that a sequence $\left\{T_{k} ; k=1,2, \ldots\right\}$ of functions converges continuously to $T$ if $\lim x_{k}=x$ and $x_{k} \in D\left(T_{k}\right)$, then $\lim T_{k} x_{k}=T x$. (There is a slight difference from Definition 6.2, but it is easy to see that they are equivalent in the case of sequences.)

Proposition 10.4. A sequence $\left\{T_{k} ; k=1,2, \ldots\right\}$ converges continuously to $T$ if and only if $\left\{T_{k}\right\}$ converges to $T$ in the compact-open topology.

Proof. The "if" part. Let $\lim x_{k}=x$. If $\left\{T_{k} x_{k}\right\}$ does not converge to $T x$ then there exists an open neighborhood $Q$ of $T x$, and a subsequence $\left\{T_{l} x_{l}\right\}$ such that $T_{l} x_{l} \notin Q$ for every $l$. The continuity of $T$ implies that for $l$ large enough, say $l \geqslant l_{0}, T x_{l} \in Q$. Since $\left\{x_{k}\right\}$ is a sequence the set $C=\left\{x_{l}\right.$ : $\left.l \geqslant l_{0}\right\} \cup\{x\}$ is compact. Although $T C \subset Q$, for every $l \geqslant l_{0}$ the relation $T_{l} C \subset Q$ does not hold. Therefore $\left\{T_{l}\right\}$ (hence $\left\{T_{k}\right\}$ ) does not converge to $T$ in the compact-open topology. The "only if" part: If $\left\{T_{k}\right\}$ converges continuously to $T$ then it is easy to see that $\left\{T_{k} x\right\}$ converges to $T x$ uniformly for $x$ in a compact set $C$. Therefore, if $Q$ is an open set that contains the compact set $T C$, then for $k$ large enough $T_{k} C \subset Q$. This completes the proof.

11. Demicompact operators. Let $X$ be a topological vector space. If $T$ is a single-valued operator then $T^{-1}$ will denote the (in general, multivalued) operator $T^{-1} y=\{x: T x=y\}$.

A single-valued operator $T$ from $X$ to itself is demicompact if whenever $\left\{x_{k}-T x_{k}\right\}$ converges and $\left\{x_{k}\right\}$ is contained in a bounded set of $X$, then the net $\left\{x_{k}\right\}$ has a convergent subnet.

The definition of demicompactness was introduced by Petryshyn [1966], for operators in a Banach space. The concept was introduced in order to discuss fixed points, i.e., solving the equation $x=T x$, or $x=T x+z$, which are the operator equations of the second kind that we discuss in this paper. For more details and references on the subject see Opial [1967]. The demicompactness of an operator is not as restrictive as it seems. For instance each of the following conditions imply that $T$ is demicompact, see Petryshyn [1966] and Opial [1967].

(a) $T$ is compact.

(b) The range of $T$ is closed, the inverse $T^{-1}$

exists and is continuous. 
(c) $X$ is Hilbert and for any $x, y$ in $D(T)$ the following inequality holds:

$$
\operatorname{Re}(T x-T y, x-y) \leqslant \frac{1}{2}\|x-y\|^{2}
$$

(d) $X$ is Hilbert and for any $x, y$ in $D(T)$ the following inequality holds:

$$
\operatorname{Re}(T x-T y, x-y) \leqslant \frac{1}{2}\|T x-T y\|^{2}
$$

We have the following characterization of demicompactness for closed operators.

Proposition 11.1. A single-valued closed operator $T$ is demicompact if and only if for every closed bounded set $B$ the operator $y \rightarrow B \cap(I-T)^{-1} y$ is compact-valued and upper semicontinuous.

Proof. Suppose $T$ is demicompact. Let $\left\{y_{k}\right\}$ converge to $y$ and $x_{k} \in B \cap$ $(I-T)^{-1} y_{k}$. If $\left\{x_{k}\right\}$ does not converge to $B \cap(I-T)^{-1} y$ then the latter is contained in an open set $Q$ such that $x_{l} \notin Q$ for a subnet $\left\{x_{l}\right\}$ of $\left\{x_{k}\right\}$. But the demicompactness implies that a subnet $\left\{x_{m}\right\}$ of $\left\{x_{l}\right\}$ converges to a certain element $x$, and the closedness of $T$ (hence of $I-T)$ implies that ( $x$, $y) \in \operatorname{Graph}(I-T)$, i.e., $x \in B \cap(I-T)^{-1} y$. Therefore $x_{m} \in Q$ for $m$ large enough, a contradiction to the construction of $\left\{x_{l}\right\}$. The demicompactness also shows that if $\left\{x_{k}\right\}$ is a net in $B \cap(I-T)^{-1} y$ then it has a converging subnet, and the closedness of $T$ implies that the limit is also in

$$
B \cap(I-T)^{-1} y \text {. }
$$

Thus $B \cap(I-T)^{-1} y$ is compact. This completes the "only if" part. In order to verify the "if" part let $\left\{y_{k}=(I-T) x_{k}\right\}$ be a converging net and assume that $\left\{x_{k}\right\}$ is included in a closed bounded set $B$. The upper semicontinuity of $y \rightarrow B \cap(I-T)^{-1} y$ implies that $\left\{x_{k}\right\}$ converges to the set

$$
B \cap(I-T)^{-1} y_{0}
$$

where $y_{0}=\lim y_{k}$. The compactness of $B \cap(I-T)^{-1} y_{0}$ implies that a subnet of $\left\{x_{k}\right\}$ converges to an element $x \in B \cap(I-T)^{-1} y_{0}$. Therefore $T$ is demicompact. This completes the proof.

The following definition is suggested by the last proposition.

Definition 11.2. A collection $\mathscr{Q}$ of closed operators is collectively demicompact if for every closed bounded set $B$ the family of operators $y \rightarrow B \cap$ $(I-T)^{-1} y$ (for $T \in \mathscr{Q}$ ) is dominated by a compact-valued upper semicontinuous mapping, i.e., there exists a compact-valued upper semicontinuous 
mapping $\Phi$ such that $\Phi(y)$ contains $B \cap(I-T)^{-1} y$ for every $T \in \mathcal{Q}$.

DEFINITION 11.3. The net $\left\{T_{k}\right\}$ of multivalued operators converges in UCC to $T$ if whenever $T_{l}$ is a subnet, $x_{l} \in D\left(T_{l}\right), \lim x_{l}=x$ and $y_{l} \in T_{l} x_{l}$, then the net $\left\{y_{l}\right\}$ converges to the set $T x$. (Compare Definition 6.2.)

By a technique similar to the proof of Theorem 6.4 we can show the following:

THEOREM 11.4. Let $\Phi$ be an upper semicontinuous compact-valued mapping from $X$ to itself. Let $थ$ be a collection of closed operators, all dominated by $\Phi$ (i.e., $T \in \mathcal{Q} \Rightarrow T y \subset \Phi(y))$. Then the Lum convergence on $\mathcal{U}$ is identical with the UCC convergence.

We omit the proof.

THEOREM 11.5 Suppose that $X$ is locally bounded. Let $\mathscr{Q}$ be a collectively demicompact family of closed operators from $X$ to itself. Then Lum $T_{k}=T$ in $\mathcal{Q}$ if and only if for every closed bounded set $B$ the operators $\{B \cap(I-$ $\left.\left.T_{k}\right)^{-1}\right\}$ converge in UCC to $B \cap(I-T)^{-1}$.

Proof. Lum $T_{k}=T$ if and only if $\operatorname{Lum}\left(I-T_{k}\right)=I-T$ (Lemma 7.2). The equality Graph $S^{-1}=\{(y, x):(x, y) \in$ Graph $S\}$ implies that

$$
\operatorname{Lum}\left(I-T_{k}\right)=I-T
$$

if and only if $\operatorname{Lum}\left(I-T_{k}\right)^{-1}=(I-T)^{-1}$. Since $X$ is locally bounded it follows that $\operatorname{Lum}\left(I-T_{k}\right)^{-1}=(I-T)^{-1}$ if and only if for every set $B$ which is closed and bounded $\operatorname{Lum} B \cap\left(I-T_{k}\right)^{-1}=B \cap(I-T)^{-1}$. Since $\mathcal{Q}$ is collectively demicompact it follows from Theorem 11.4 that the last equality is equivalent to the convergence in UCC of $\left\{B \cap\left(I-T_{k}\right)^{-1}\right\}$ to $B \cap(I-$ $T)^{-1}$. This completes the proof.

As was noted before, the concept of demicompactness was introduced by Petryshyn in connection with his study of fixed points, and thus, in general, with solutions of the equation $x=T x+z$. We shall describe now the implication of the previous results in this section to the continuous dependence of the solutions set. We again denote by $s(T, z)$ the set of solutions of the operator equation.

Proposition 11.6. If $T$ is closed and demicompact then for every closed and bounded set $B$ the set $B \cap s(T, z)$ is compact.

Proof. Immediate from Proposition 11.1 and the equality $s(T, z)=$ $(I-T)^{-1} z$.

TheOREM 11.7. Suppose that $X$ is locally bounded. Let $\mathscr{Q}$ be a collectively demicompact family of closed operators. The convergence on $\mathcal{Q}$ determined by UCC convergence of $B \cap(I-T)^{-1}$ for every closed bounded $B$ is the richest 
convergence structure on o with respect to which for every such $B$ the multivalued mapping $B \cap s(T, z)$ is upper semicontinuous.

Proof. By Theorem 11.4 the convergence described in the statement is the Lum convergence, and for $L u$-continuity the result is given by Theorem 5.3. Since $s(T, z)=(I-T)^{-1} z$, it follows that $s(T, z) \cap B$ is dominated by a compact-valued upper semicontinuous mapping, and therefore its $\mathrm{Lu}$-continuity is equivalent to upper semicontinuity.

\section{REFERENCES}

P. M. Anselone [1971], Collectively compact operator approximation theory, Prentice-Hall, Englewood Cliffs, N. J.

R. Arens [1946], A topology for spaces of transformations, Ann. of Math. (2) 47, 480-495. MR 8, 165.

Z. Artstein [1975a], Continuous dependence on parameters: On the best possible results, J. Differential Equations 19, 214-225.

[1975b], Continuous dependence of solutions of Volterra integral equations, SIAM J. Math. Anal. 6, 446-456. MR 50 \# 14101.

[1976], On continuous dependence of fixed points of condensing maps, Dynamical

Systems, Vol. III, An international symposium, Academic Press, New York, pp. 73-75.

E. Čech [1966], Topological spaces, 2nd ed., Publ. House Czech. Acad. Sci., Prague; English transl., Interscience, New York. MR 21 \#2962; 35 \#2254.

T. Costello [1971], On the fundamental theory of functional-differential equations, Funkcial. Ekvac. 14, 177-190. MR 46 \#5796.

G. Darbo [1955], Punti uniti in trasformazioni a codominio non compatto, Rend. Sem. Mat. Univ. Padova 24, 84-92. MR 16, 1140.

R. D. Driver [1965], Existence and continuous dependence of solutions of a neutral functional-differential equation, Arch. Rational Mech. Anal. 19, 149-166. MR 31 \#3654.

R. Gaines [1969], Continuous dependence for two-point boundary value problems, Pacific J. Math. 28, 327-336. MR 39 \# 1728.

L. J. Grimm [1971], Existence and continuous dependence for a class of nonlinear neutral-differential equations, Proc. Amer. Math. Soc. 29, 467-473. MR 44 \# 4324.

J. K. Hale [1973], $\alpha$ contraction and differential equations, Proc. Equations Differential Fon. Nonlin. (Brussels, 1973), Hermann, Paris, pp. 15-42.

46, 388-393. MR 50 \#5559.

[1974], Continuous dependence of fixed points of condensing maps, J. Math. Anal. Appl.

J. K. Hale and M. A. Cruz [1970], Existence, uniqueness and continuous dependence for hereditary systems, Ann. Mat. Pura Appl. (4) 85, 63-81. MR 41 \# 7238.

S. K. Ingram [1972], Continuous dependence on parameters and boundary data for nonlinear two-point boundary value problems, Pacific J. Math. 41, 395-408. MR 46 \#3873.

J. L. Kelley [1955], General topology, Van Nostrand, Princeton, N. J. MR 16, 1136.

G. Klaasen [1975], Continuous dependence for $N$-point boundary value problems, SIAM J. Appl. Math. 29, 99-102.

G. Köthe [1969], Topological vector spaces. I, 2nd. cor. ed., Springer-Verlag, New York. MR 33 \#3069; 40 \#1750.

K. Kuratowski [1930], Sur les espaces completes, Fund. Math. 15, 301-309. [1966], Topology. I, 5th ed., PWN, Warsaw; Academic Press, New York. MR 36 \#840.

J. Kurzweil and Z. Vorel [1957], Continuous dependence of solutions of differential equations on a parameter, Czechoslovak Math. J. 7 (82), 568-583. MR 22 \#2734.

L. W. Neustadt [1970], On the solutions of certain integral-like operator equations. Existence, uniqueness and dependence theorems, Arch. Rational Mech. Anal. 38, 131-152. MR 41 \#7498. 
Z. Opial [1967], Nonexpansive and monotone mappings in Banach spaces, Lecture notes 67-1, Center for Dynamical Systems, Brown Univ., Providence, R. I.

W. V. Petryshyn [1966], Construction of fixed points of demicompact mappings in Hilbert space, J. Math. Anal. Appl. 14, 274-284. MR 33 \#3147.

S. Szufla [1971], Measure of non-compactness and ordinary differential equations in Banach spaces, Bull. Acad. Polon. Sci. Sér. Sci. Math. Astronom. Phys. 19, 831-835. MR 46 \#2185.

T. Wenska [1972], Imbedding of nonlinear integral equations, J. Math. Anal. Appl. 39, 670-678. MR 47 \# 739.

Lefschetz Center for Dynamical Systems, Division of Applied Mathematics, Brown UNIVERSity, Providence, RHOde Island 02912

Department of Mathematics, The Weizmann Institute of Science, Rehovot, Israel 